\title{
Estudo da produção científica da revista Psico-USF de 2007 a 2011
}

\author{
Lariana Paula Pinto1 - Universidade São Francisco, Itatiba, Brasil \\ Thatiana Helena de Lima - Universidade São Francisco, Itatiba, Brasil \\ Robisom Carlos de Lima - Universidade São Francisco, Itatiba, Brasil
}

\begin{abstract}
Resumo
O presente estudo investigou a produção científica da revista Psico-USF, entre os anos de 2007 a 2011. Foram utilizados 12 volumes, dos quais 155 artigos foram analisados com base nos seguintes critérios: quantidade de artigos publicados por número da revista, região em que prevaleceram as pesquisas, natureza da autoria, gênero dos autores, referências utilizadas na fundamentação dos artigos publicados, distribuição por tema de estudo e por tipo de trabalho, bem como por tipo de material utilizado nas pesquisas de campo, e as referências utilizadas. Os dados evidenciaram a manutenção da média de artigos publicados por número, dando destaque, entretanto, para o acréscimo de um terceiro número de publicação ao ano. Observou-se que o relato de pesquisa foi a forma mais utilizada e a região sudeste, a que mais publicou nesse período. Houve ainda a predominância da autoria múltipla e feminina e, quanto às temáticas, a maioria dos trabalhos se voltou aos fundamentos e medidas em psicologia. Quanto às referências utilizadas, a maioria dos trabalhos se fundamentou preponderantemente em artigos de periódicos científicos.

Palavras-chave: Produção científica, Psicologia, Periódicos, Qualidade das publicações.
\end{abstract}

\section{Science production of Psico-USF journal: from 2007 to 2011}

\begin{abstract}
The present study investigated the scientific production of PsicoUSF journal between the years 2007 to 2011.12 volumes were used, in which 155 articles were analyzed based in the following criteria: number of articles published per issue of the journal, region that prevailed at the polls, nature of authorship, gender of authors, references used in the grounds of published articles, distribution by subject of study and type of work as well as by type of material used in field research and the references used. The data showed the maintenance of the mean number of articles published per issue, highlighting, however, for the addition of a third number of publications per year. It was observed that the research report was the most used and the Southeast, the most published during this period. There was also predominance of multiple authorship and female, and about the issues, most of the work turned to the Fundamentals and measures in psychology. As for the references used, most studies relied primarily on journal articles.

Keywords: Science production, Psychology, Journals, Quality of publications.
\end{abstract}

Sendo considerados indicadores e parâmetros de mensuração e caminho para o desenvolvimento da ciência, a quantidade e a qualidade da produção científica são aspectos centrais em qualquer área do conhecimento. Dessa forma, a divulgação da ciência é considerada um aspecto crucial para o seu progresso, dada à sua importância no cenário mundial, bem como o papel de destaque da comunicação (Yamamoto, Souza \& Yamamoto, 1999; Soares, Victoria, Cavalieri \& Bottino, 2006). De acordo com Freitas (2006), além de retratar historicamente os principais fatos do desenvolvimento da ciência, o periódico científico pode ser considerado um espaço institucional, pois se insere no universo das realizações e comunicação formal das atividades científicas.

No Brasil, a primeira revista de psicologia editada foi a Revista Brasileira de Psicanálise, com o primeiro número publicado em 1928. Desde então, outros periódicos científicos foram editados na área, tais como a revista Boletim de Psicologia, editada em 1945,

\footnotetext{
${ }^{1}$ Endereço para correspondência:

Av. Barão do Rio Branco, 600 - Centro

Conceição dos Ouros - 37548-000 - MG

E-mail: lariana_paula@hotmail.com
}

com o objetivo de promover o desenvolvimento da psicologia como ciência e profissão, e a revista Arquivos Brasileiros de Psicotécnica, em 1949. A partir daí, um grande número de títulos que publicam sobre psicologia surgiu, sendo difícil, atualmente, precisar o número exato de periódicos de psicologia (Conselho Regional de Psicologia de São Paulo, 2007; Gonçalves, Ramos \& Castro, 2006, citados por Suehiro \& Rueda, 2009). Ainda, Hill (2004) menciona que houve aumento expressivo das publicações realizadas por pesquisadores latino-americanos, com especial destaque para os brasileiros, cujos trabalhos representaram acréscimo de quatro vezes no período de 1988 a 2001.

Nesse rol de revistas, encontra-se a revista Psico-USF, vinculada ao Programa de Pós-Graduação Stricto Sensu em Psicologia da Universidade São Francisco, com publicação quadrimestral. De acordo com Suehiro, Cunha, Oliveira e Pacanaro (2007), é editada desde 1974, já tendo sido denominada Klínica e Revista das Faculdades Franciscanas, tendo recebido o nome atual em 1996. Ela destina-se a publicar trabalhos originais que relatem estudos em áreas relacionadas à psicologia, incluindo processos básicos, experimentais, aplicados, naturalísticos, etnográficos, históricos, artigos teóricos, análises de políticas e sínteses 
sistemáticas de pesquisas, entre outros, assim como revisões críticas de livros, instrumentos diagnósticos e softwares. Como processo editorial, a revista conta com uma revisão às cegas de pareceristas ad hoc, permitindo que, em consequência, seus conteúdos não reflitam a posição, opinião ou filosofia do Programa de PósGraduação nem da Universidade São Francisco, nos quais está inserida (Suehiro e cols., 2007; Periódicos Eletrônicos em Psicologia, 2011).

Ainda em relação à Psico-USF, com o objetivo de criar um elo de comunicação entre os pesquisadores da Universidade São Francisco (USF) e os de outras instituições de ensino, a revista compromete-se a possibilitar aos pesquisadores da USF um diálogo científico nacional, considerando ser este uma exigência sine qua non para melhorar a qualidade do ensino, além de que os estudos e trabalhos fossem originais e relevantes para a área da psicologia, conforme apontam Suehiro e cols. (2007).

Inserida, portanto, no grupo dos periódicos nacionais, a revista está atualmente classificada como B1, de acordo com a avaliação da Qualis, que, por sua vez, diz respeito à estratificação da qualidade da produção intelectual dos programas de pós-graduação, que subsidia a avaliação dos programas de pósgraduação credenciados pela instituição e é alimentada a partir dos relatórios enviados pelos referidos programas, conduzida pela Coordenação de Aperfeiçoamento de Pessoal de Nível Superior CAPES e a estreita colaboração da Associação Nacional de Pesquisa e Pós-Graduação em Psicologia ANPEPP (Yamamoto e cols., 2002).

Yamamoto e cols. (2002), ao analisarem a produção dos periódicos científicos brasileiros da área da psicologia, puderam evidenciar a perceptível melhora na classificação das revistas nacionais. Em 1999 houve a realização inicial do empreendimento, cujas demandas foram a qualidade dos periódicos em que os pesquisadores dos programas de pós-graduação em psicologia publicavam; a facilitação, por meio de novos mecanismos, com fins de melhorar a avaliação; e determinar critérios para o desenvolvimento da qualidade das revistas da área.

De certa forma, esses veículos de comunicação acabam por ser submetidos a avaliações de sua produção científica tanto por pesquisadores quanto por instituições, ao analisarem, por exemplo, indicadores de citação, autoria, co-autoria e acesso, entre outros. Isso porque a avaliação dos periódicos está entre as estratégias que podem favorecer um modelo de qualidade nas publicações e avanços no campo do conhecimento (Witter, 1999; Yamamoto, Souza \& Yamamoto, 1999; Yamamoto e cols. 2002).
Nessa linha, alguns estudos já foram realizados com o objetivo de analisar determinadas características das publicações nos periódicos, inclusive tendo como marcação cronológica períodos diferentes. Entre eles pode ser citado o de Yamamoto, Souza e Yamamoto (1999), que analisaram seis periódicos de psicologia, de circulação nacional, quanto aos aspectos, política de publicação, perfis dos autores e as instituições às quais pertenciam. Investigaram 749 artigos distribuídos pelas 80 edições dos periódicos. Os resultados mostraram que poucos autores, no Brasil, publicam sistematicamente. As publicações estão concentradas em instituições das regiões sul e sudeste e que as universidades mais produtivas são as publicas, quando comparadas às particulares.

A pesquisa realizada por Oliveira, Cantalice, Joly e Santos (2006) objetivou a investigação da produção científica dos últimos dez anos da Revista de Psicologia Escolar e Educacional. Para tanto, foram analisados os critérios, autoria, temática, discurso e análise dos tipos de avaliações. Dentre os resultados, as autoras encontraram que os volumes publicados nos cinco últimos anos apresentavam mais artigos publicados. A pesquisa de campo foi a que teve mais publicações e a região sudeste obteve maior número de artigos. Quanto aos tipos de autorias, foi predominante a múltipla e feminina. Houve uma diversificação das temáticas e dentro da área escolar, o nível mais estudado foi o superior e o menos, foi o médio. As autoras pontuaram um amadurecimento da área, mas que alguns pontos ainda poderiam ser mais explorados.

Outro estudo que se propôs a pesquisar a produção de revista, dessa vez, da revista Psico-USF, foram Suehiro e cols. (2007). Para análise foram consultados 11 volumes, totalizando 188 artigos, com base nos seguintes critérios: quantidade, média de volumes e artigos publicados por ano, tipo de trabalho, região de origem, natureza da autoria, gênero dos autores, temas de avaliação, palavras do título, amostra e etapa de escolarização. Dentre os resultados obtidos, as autoras evidenciaram um aumento as publicações nos últimos seis anos, sendo o ano de 2006 o de maior publicação. Como no estudo anterior, o relato de pesquisa foi o tipo de trabalho mais publicado e a região sudeste a que mais publicou. Da mesma forma, houve maioria de autoria múltipla e predominância do sexo feminino. A temática mais publicada é a de fundamentos e medida em psicologia. $O$ estudo, segundo as autoras, trouxe uma vasta exposição dos aspectos pesquisados, assim como de temas que ainda precisarim ser explorados.

O objetivo da pesquisa de Suehiro e Rueda (2009) foi analisar as publicações da revista Avaliação Psicológica. Foram analisados 12 números, com total de 
100 artigos, quanto aos aspectos, quantidade de artigos publicados por número da revista, distribuição da produção por origem, natureza da autoria, gênero dos autores, tipo de trabalho, tipo de material utilizado nas pesquisas de campo e referências empregadas na fundamentação dos artigos. Os resultados mostraram que houve aumento das publicações nos últimos anos avaliados e o ano de 2007 foi o de maior publicação. Como nos dois estudos descritos anteriormente, o relato de pesquisa foi o mais publicado, assim como a região sudeste a que mais publicou. Quanto ao tipo de autoria, a maioria foi múltipla e do sexo feminino. Os artigos de periódicos científicos foram base para a maioria dos estudos, e isso era esperado pelos autores.

Ainda em 2009, Rueda analisou a produção científica da Revista Brasileira de Orientação Profissional. O autor examinou 12 números, totalizando 81 artigos, e foram utilizados os seguintes critérios, quantidade de artigos publicados por número da revista, distribuição da produção por origem, natureza da autoria, sexo dos autores, sexo do primeiro autor, tipo de trabalho, instrumentos e referências utilizadas na fundamentação dos artigos. Dentre os resultados, percebeu-se um aumento de publicações no ano de 2008. Corroborando dados anteriores, o relato de pesquisa foi o mais publicado, assim como a predominância de publicação da região sudeste, da autoria múltipla e feminina. Quanto às referências, as fundamentações dos trabalhos foram de livros ou capítulos de livros e artigos de periódicos científicos.

Considerando o exposto até aqui, é evidente a importância de se analisar e avaliar a produção científica, que por sua vez pode revelar o status quo da ciência e do conhecimento, nas suas áreas específicas, bem como o desenvolvimento e o aprimoramento das mesmas. Sendo assim, o presente estudo teve como objetivo analisar a tendência das publicações efetivadas na revista Psico-USF no período de 2007 a 2011, dando continuidade ao trabalho publicado por Suehiro e cols. (2007).

\section{Método}

Fonte

Foram analisados 11 números da revista PsicoUSF, cujas publicações totalizaram 155 artigos e 24 resenhas. Para o presente estudo somente os artigos foram analisados. Para a realização do levantamento, foram utilizadas as versões impressas do periódico.

\section{Procedimento}

Os artigos foram analisados na íntegra, tomando como base alguns critérios estabelecidos por Witter (1999) e outros considerados relevantes pelos autores. Os itens pesquisados foram: (a) a quantidade de artigos publicados por número da revista; (b) a distribuição da produção por origem (Sul, Sudeste, Centro-Oeste, Nordeste, Norte, parcerias regionais, internacionais e parcerias nacionais e internacionais); (c) a natureza da autoria (individual ou múltipla); (d) o gênero dos autores; (e) as referências utilizadas em cada artigo publicado; (f) a distribuição por temas de estudo; (g) a distribuição do tipo de trabalho (relato de pesquisa de campo, pesquisa documental, ou manuscrito teórico); e (h) nas pesquisas de campo procurou-se pesquisar, ainda, o tipo de material utilizado (testes ou escalas, podendo ser eles nacionais, internacionais ou traduzidos para o português; aqueles artigos que utilizaram outro tipo de material, tais como observações, questionários elaborados pelo(s) autor(es) ou outro tipo de procedimento específico; e ainda, os trabalhos mistos, caracterizados pela utilização de instrumentos de medida e outro tipo de material).

\section{Resultados e discussão}

Para a realização das análises das publicações da Psico-USF, foram levantados a quantidade de volumes, números e o total de artigos e comunicações breves publicados por ano. Esses primeiros resultados são apresentados na Tabela 1.

A partir da Tabela 1 foi possível observar que a média de artigos publicados por número $(M=12,92)$ foi mantida ao longo dos anos. Entretanto, visto que a partir de 2009 a revista passou a apresentar três números ao ano/volume, pode-se afirmar que corresponde ao aumento de número de produções. Esse crescimento também foi apontado por Suehiro e cols. (2007), que ao realizarem levantamento da produção científica da revista Psico-USF verificaram aumento no número das publicações nos últimos seis anos. Outros autores também apontam para o desenvolvimento das publicações, tanto no âmbito nacional como no internacional (Hill, 2004; Oliveira e cols., 2006; Yamamoto \& Gouveia, 2003). 
Tabela 1. Distribuição geral da quantidade de volumes e número, bem como do total de artigos publicados por ano $(\mathrm{N}=155)$

\begin{tabular}{|c|c|c|c|c|}
\hline Ano & Volume & Número & Total de artigos por número & Total de publicações por ano \\
\hline \multirow{2}{*}{2007} & \multirow{2}{*}{12} & 1 & 13 & \multirow{2}{*}{33} \\
\hline & & 2 & 20 & \\
\hline \multirow{2}{*}{2008} & \multirow{2}{*}{13} & 1 & 14 & \multirow{2}{*}{28} \\
\hline & & 2 & 14 & \\
\hline \multirow{3}{*}{2009} & \multirow{3}{*}{14} & 1 & 11 & \multirow{3}{*}{34} \\
\hline & & 2 & 11 & \\
\hline & & 3 & 12 & \\
\hline \multirow{3}{*}{2010} & \multirow{3}{*}{15} & 1 & 12 & \multirow{3}{*}{36} \\
\hline & & 2 & 12 & \\
\hline & & 3 & 12 & \\
\hline \multirow{2}{*}{2011} & \multirow{2}{*}{16} & 1 & 12 & \multirow{2}{*}{24} \\
\hline & & 2 & 12 & \\
\hline
\end{tabular}

Para avaliar a origem da produção, levantou-se a procedência da autoria dos artigos. Assim, avaliou-se a região do Brasil, o país e as parcerias regionais e internacionais realizadas. Cabe ressaltar que foram consideradas parcerias os casos em que os manuscritos tenham sido elaborados por autores de regiões ou países diferentes; e quando os articulistas eram provenientes de diferentes instituições, porém da mesma região, foi considerada a região de ambos. Por fim, foram considerados artigos internacionais aqueles que continham autores do mesmo país de origem estrangeira ou autores de mais de um país estrangeiro. Os resultados são apresentados na Tabela 2.

Tabela 2. Distribuição da produção por origem $(\mathrm{N}=155)$

\begin{tabular}{lcc}
\hline Origem & F & $\%$ \\
\hline Sul & 30 & 19,35 \\
Sudeste & 60 & 38,71 \\
Centro-Oeste & 10 & 6,45 \\
Norte & 1 & 0,65 \\
Nordeste & 11 & 7,10 \\
Parceria Nacional & 23 & 14,84 \\
Parceria Internacional & 7 & 4,52 \\
Internacional & 13 & 8,39 \\
Total & 155 & 100 \\
\hline
\end{tabular}

A Tabela 2 evidencia que, no que se refere à origem da produção, a região Sudeste foi a que mais contribuiu para as publicações da revista, representando 38,71\% ( $\mathrm{n}=60)$ do total de publicações dos quatro anos levantados, seguida da região Sul $(\mathrm{n}=30 ; 19,35 \%)$, ao passo que a região Norte contribuiu com apenas uma publicação $(0,65 \%)$. O fato de a região Sudeste ter apresentado maior quantidade de publicações tem ecoado em outros estudos, como é o caso de Oliveira e cols. (2006), Soares e cols. (2006), Suehiro e cols. (2007), Suehiro e Rueda (2009), e Yamamoto e cols. (1999).

No que se refere à natureza da autoria, individual ou múltipla, verificou-se que os artigos foram escritos, em sua maioria, por mais de um autor ( $n=148 ; 70,89 \%$ ), assim como observado por Oliveira e cols. (2006) e Suehiro e cols. (2007). Quanto ao gênero dos autores, dos 426 que publicaram artigos, individual ou coletivamente, a maior parte foi do sexo feminino ( $n=302 ; 71,79 \%)$, e quando publicados coletivamente, em $112(75,67 \%)$ deles o primeiro autor foi do sexo feminino. É marcante o predomínio do sexo feminino na autoria dos trabalhos que têm sido divulgados por periódicos científicos, como verificado por diversos estudos, dentro os quais o de Yamamoto e cols. (1999), Oliveira e cols. (2006), Suehiro e cols. (2007) e Suehiro e Rueda (2009). 
As referências utilizadas nas publicações também foram analisadas. Para tanto, foram classificadas em seis categorias, quais sejam, (a) artigo publicado, no prelo ou submetido para publicação; (b) livro ou capítulo de livro; (c) tese de doutorado, dissertação de mestrado, relatório técnico, monografia de especialização ou trabalho de conclusão de curso; (d) manual de teste; (e) trabalho apresentado em congresso, podendo o mesmo ser na forma de comunicação oral, painel, mesa-redonda ou conferência e; (f) categoria outro, sendo agrupadas nesta categoria as leis, resoluções e manuscritos não-publicados. Os resultados dessa análise podem ser observados na Tabela 3.

Tabela 3. Tipos de referências utilizadas em cada número da revista Psico-USF

\begin{tabular}{|c|c|c|c|c|c|c|c|c|c|c|c|c|c|c|c|}
\hline \multirow[t]{2}{*}{ Ano } & \multirow[t]{2}{*}{ Vol. } & \multirow[t]{2}{*}{ Núm. } & \multicolumn{2}{|c|}{$\begin{array}{c}\text { Artigo } \\
\text { publicado, } \\
\text { no prelo ou } \\
\text { submetido }\end{array}$} & \multicolumn{2}{|c|}{$\begin{array}{l}\text { Livro ou } \\
\text { capítulo de } \\
\text { livro }\end{array}$} & \multicolumn{2}{|c|}{$\begin{array}{c}\text { Tese, } \\
\text { Dissertação, } \\
\text { Relatório } \\
\text { técnico, } \\
\text { Monografia } \\
\text { ou trabalho } \\
\text { de } \\
\text { conclusão } \\
\text { de curso }\end{array}$} & \multicolumn{2}{|c|}{$\begin{array}{l}\text { Manual } \\
\text { de teste }\end{array}$} & \multicolumn{2}{|c|}{$\begin{array}{c}\text { Trabalho } \\
\text { apresentado } \\
\text { em congresso } \\
\text { (comunicação } \\
\text { oral, painel, } \\
\text { mesa- } \\
\text { redonda, } \\
\text { conferência, } \\
\text { etc.) }\end{array}$} & \multicolumn{2}{|c|}{ Outro } & \multirow[t]{2}{*}{$\begin{array}{c}\text { Total } \\
\\
\frac{f}{}\end{array}$} \\
\hline & & & $\bar{f}$ & $\%$ & $\bar{f}$ & $\%$ & $\bar{f}$ & $\%$ & $f$ & $\%$ & $f$ & $\%$ & $F$ & $\%$ & \\
\hline \multirow{2}{*}{2007} & \multirow{2}{*}{12} & 1 & 196 & 59,76 & 94 & 28,66 & 10 & 3,05 & 11 & 3,35 & 7 & 2,13 & 10 & 3,05 & 328 \\
\hline & & 2 & 310 & 47,91 & 210 & 32,46 & 37 & 5,72 & 27 & 4,17 & 37 & 5,72 & 26 & 4,02 & 647 \\
\hline \multirow{3}{*}{2008} & \multirow{3}{*}{13} & 1 & 318 & 65,84 & 125 & 25,88 & 21 & 4,35 & 9 & 1,86 & 1 & 0,21 & 9 & 1,86 & 483 \\
\hline & & 2 & 218 & 50,23 & 161 & 37,10 & 31 & 7,14 & 6 & 1,38 & 7 & 1,61 & 11 & 2,53 & 434 \\
\hline & & 1 & 175 & 51,17 & 126 & 36,84 & 17 & 4,97 & 6 & 1,75 & 8 & 2,34 & 10 & 2,92 & 342 \\
\hline \multirow[t]{3}{*}{2009} & \multirow[t]{3}{*}{14} & 2 & 162 & 50,47 & 129 & 40,19 & 10 & 3,12 & 10 & 3,12 & 3 & 0,93 & 7 & 2,18 & 321 \\
\hline & & 3 & 180 & 56,78 & 100 & 31,55 & 17 & 5,36 & 9 & 2,84 & 7 & 2,21 & 4 & 1,26 & 317 \\
\hline & & 1 & 220 & 62,50 & 113 & 32,10 & 11 & 3,13 & 2 & 0,57 & 2 & 0,57 & 4 & 1,14 & 352 \\
\hline \multirow[t]{2}{*}{2010} & \multirow[t]{2}{*}{15} & 2 & 225 & 60,98 & 103 & 27,91 & 19 & 5,15 & 9 & 2,44 & 4 & 1,08 & 9 & 2,44 & 369 \\
\hline & & 3 & 251 & 62,28 & 116 & 28,78 & 18 & 4,47 & 5 & 1,24 & 6 & 1,49 & 7 & 1,74 & 403 \\
\hline \multirow{2}{*}{2011} & \multirow{2}{*}{16} & 1 & 254 & 57,86 & 128 & 29,16 & 20 & 4,56 & 23 & 5,24 & 12 & 2,73 & 2 & 0,46 & 439 \\
\hline & & 2 & 160 & 48,34 & 118 & 35,65 & 18 & 5,44 & 13 & 3,93 & 6 & 1,81 & 16 & 4,83 & 331 \\
\hline Total & 6 & 12 & 2669 & 56 & 1523 & 31,96 & 229 & 4,80 & 130 & 2,73 & 100 & 2,10 & 115 & 2,41 & 4766 \\
\hline
\end{tabular}

Pela Tabela 3 pode ser verificado que em todos os números da revista Psico-USF houve maior consulta a artigos científicos, sendo que, do total de referências utilizadas em todos os artigos publicados no período de 2007 a 2011, esse tipo de citação correspondeu a $56 \%$. Os livros ou capítulos de livro encontram-se como segundo tipo de fonte utilizado, apresentando média de 31,96\% entre todas as referências. Tais resultados estão de acordo com os obtidos por Suehiro e cols. (2007) e Suehiro e Rueda (2009), que constataram que as informações mais utilizadas pelos autores para a fundamentação dos artigos científicos analisados foram aquelas veiculadas ou divulgadas pelos periódicos científicos.

Também se buscou analisar a distribuição das publicações por temáticas pesquisadas, que foram consideradas de acordo com a listagem de áreas do conhecimento disponibilizada pela Plataforma Lattes do Conselho Nacional de Desenvolvimento Científico e Tecnológico (CNPq). A distribuição de tais temáticas pode ser visualizada na Tabela 4. 
Tabela 4. Frequências referentes à formação dos autores (N=155)

\begin{tabular}{lcc}
\hline \multicolumn{1}{c}{ Temáticas } & F & $\%$ \\
\hline Fundamentos e medidas da psicologia & 70 & 45,16 \\
Desenvolvimento humano & 6 & 3,87 \\
Ensino e aprendizagem & 6 & 3,87 \\
Psicologia organizacional e do trabalho & 6 & 3,87 \\
Psicologia experimental & 1 & 0,65 \\
Psicologia social & 4 & 2,58 \\
Tratamento e prevenção psicológica & 6 & 3,87 \\
Psicologia da saúde e hospitalar & 8 & 5,16 \\
Mais de uma temática & 25 & 16,13 \\
Outra & 23 & 14,84 \\
Total & 155 & 100 \\
\hline
\end{tabular}

Quase metade dos artigos analisados $(n=70$; $45,16 \%$ abordou assuntos relacionados a "Fundamentos e medidas da psicologia", conforme apresentado na Tabela 4, que referem-se a construção e validade de testes, escalas e outras medidas psicológicas; história, teorias e sistemas em psicologia; metodologia, instrumentação e equipamento em psicologia e técnicas de processamento estatístico, matemático e computacional em psicologia. Esses resultados também foram encontrados por Suehiro e cols. (2007).

No que diz respeito à análise por tipo de trabalho, averiguou-se a modalidade na qual o artigo enquadrava-se, podendo ser relato de pesquisa, pesquisa documental ou manuscrito teórico. Os resultados evidenciaram que a maior parte do material publicado tratava-se de relatos de pesquisa $(n=134$; $86,45 \%)$, ao passo que as pesquisas documentais $(\mathrm{n}=11,7,10 \%)$ e os manuscritos teóricos $(\mathrm{n}=10$, $6,45 \%)$ se apresentaram semelhantemente. Isso também foi observado por Oliveira e cols. (2006), Suehiro e cols. (2007) e Suehiro e Rueda (2009).

Dentre os relatos de pesquisa $(n=134)$, ainda verificou-se que tipo de material foi utilizado no desenvolvimento do estudo, sendo considerados para análise testes ou escalas, podendo ser eles nacionais, internacionais ou traduzidos para o português; outro tipo de material, tal como observações, questionários elaborados pelo(s) autor(es) ou outro procedimento específico; e, ainda, os trabalhos mistos, caracterizados pela utilização de instrumentos de medida e outro de material. Assim, verificou-se que metade dos artigos utilizou testes ou escalas ( $\mathrm{n}=68 ; 50,75 \%$ ), seguidos pelos manuscritos que utilizaram outro tipo de material $(\mathrm{n}=42 ; 31,34 \%)$ e, por fim, os trabalhos mistos $(\mathrm{n}=24$; 17,91\%). Dessa forma, também foram os achados de Oliveira e cols. (2006) e Suehiro e Rueda (2009). Essas confirmações parecem apontar para a preocupação crescente dos autores em aprimorar as propriedades e qualidades psicométricas dos instrumentos, beneficiando o desenvolvimento e aprimoramento das técnicas de mensuração.

\section{Considerações finais}

O objetivo do presente estudo foi realizar um levantamento dos trabalhos publicados na revista PsicoUSF, desde 2007 até o segundo número publicado no ano de 2011, com a intenção de apresentar um panorama de suas publicações e seu desenvolvimento. Para tanto, foram consideradas algumas categorias, quais sejam, quantidade de artigos publicados por número da revista, região em que prevaleceram as pesquisas, natureza da autoria, gênero dos autores, referências utilizadas na fundamentação dos artigos publicados, distribuição por tema de estudo e por tipo de trabalho, bem como por tipo de material utilizado nas pesquisas de campo e as referências utilizadas.

Os achados do presente estudo destacam que a revista Psico-USF manteve seu crescimento, com destaque principalmente a partir da publicação de três números ao ano, acompanhando a tendência dos periódicos dos Estados Unidos e da América Latina, conforme encontrado em revisão anterior (Hill, 2004; Suehiro e cols., 2007). Paralelamente, também confirmou-se mais uma vez a predominância da região Sudeste como a mais produtiva, assim como uma maior frequência de trabalhos com autoria múltipla e feminina.

No que diz respeito às temáticas, constatou-se que houve predominância de publicações na categoria "Fundamentos e medidas da psicologia", o que pode estar refletindo a revalorização da área pelos profissionais da psicologia, que mobilizou alguns 
segmentos da comunidade científica e profissional. A esse respeito, pode-se citar a criação do Instituto Brasileiro de Avaliação Psicológica (IBAP) e a Resolução n. ${ }^{\circ}$ 02/2003 do Conselho Federal de Psicologia (CFP), que regulamentou o uso, elaboração e comercialização de testes psicológicos no Brasil.

Ao lado disso, discussões sobre os materiais ou técnicas utilizadas também merecem destaque, uma vez que houve predominância de trabalhos que utilizaram testes ou escalas. Essa questão pode ser foco de futuras investigações, para que mais do que verificar quais instrumentos de medida estão sendo utilizados, possa ser verificado de que forma eles estão sendo usados, para que finalidade, e se apresentam as características psicométricas necessárias, já que se considera que a quantidade de instrumentos disponíveis nessa área ainda é escasso.

De maneira geral, os resultados aqui apresentados acabam por reforçar a importância de serem realizadas pesquisas recorrentes dos periódicos científicos, com o objetivo de buscar avaliar a qualidade do conteúdo publicado, propiciando a caracterização e tendências de publicações dos periódicos científicos, que podem, por sua vez, gerar diretrizes para novos temas e formatos de pesquisas, bem como a distribuição de fomentos.

Em que pesem as limitações desta pesquisa, considera-se que estudos com um maior aprofundamento devem ser realizados, levando em consideração as possibilidades de investigação citadas anteriormente, bem como a apresentação de outros dados específicos das publicações, que aqui não foram levantados. Pesquisas deste porte são relevantes não apenas por possibilitarem o desenho e o desenvolvimento dos periódicos científicos, mas, sobretudo, porque funcionam como um indicativo das lacunas que precisam ser preenchidas, contribuindo assim para a disseminação, a qualidade e o progresso do conhecimento produzido nas academias.

\section{Referências}

Coordenação de Aperfeiçoamento de Pessoal de Nível Superior (2011). Qualis Periódicos. Obtido em 13 de novembro de 2011 do World Wide Web: http://www.capes.gov.br/avaliacao/qualis.

Freitas, M. H. A. (2006). Considerações acerca dos primeiros periódicos científicos brasileiros. Ciência da Informação, 35(3), 163-177.

Hill, D. L. (2004). Latin American shows rapid rise in published Science \& Engineering articles. National Science Foundation. Obtido em 11 de novembro de
2011 do World Wide Web: http://nsf.gov/statistics/infbrief/nsf04336/nsf04 336.pdf.

Oliveira, K. L., Cantalice, L. M., Joly, M. C. A. \& Santos, A. A. A. (2006). Produção científica de 10 anos da revista Psicologia Escolar e Educacional (1996/2005). Psicologia Escolar e Educacional, 10(1), 283-292.

Periódicos Eletrônicos em Psicologia (2007). PEPSIC: Periódicos Eletrônicos em Psicologia. Universidade de São Paulo. Obtido em 13 de novembro de 2011 do World Wide Web: http://pepsic.bvs-psi.org.br.

Rueda, F. J. M. (2009). Produção científica da Revista Brasileira de Orientação Profissional. Revista Brasileira de Orientação Profissional, 10(2), 129-139.

Soares, A. B., Victoria, M. S., Cavalieri, A. M. A. P. \& Bottino, A. G. (2006). A Psicologia divulgada através de periódicos científicos indexados e através de revistas de grande circulação do Brasil: um estudo preliminar. Psicología para América Latina, $7 . \quad$ Disponível em: http://www.psicolatina.org/Siete/divulgada.html.

Suehiro, A. C. B. \& Rueda, F. J. M. (2009). Revista Avaliação Psicológica: um estudo da produção científica de 2002 a 2007. Avaliação Psicológica, 8(1), 131-139.

Suehiro, A. C. B., Cunha, N. B., Oliveira, E. Z. \& Pacanaro, S. V. (2007). Produção científica da revista Psico-USF de 1996 a 2006. Psico-USF, 12(2), 327-334.

Witter, G. P. (1999). Metaciência e leitura. Em G. P. Witter (Org.). Leitura: textos e pesquisas. (pp. 13-22). Campinas: Alínea.

Yamamoto, O. H., Souza, C. C. \& Yamamoto, M. E. (1999). A produção científica na psicologia: uma análise dos periódicos brasileiros no período de 1990-1997. Psicologia: Reflexão e Crítica, 12(2), 549565.

Yamamoto, O. H., Menandro, P. R. M., Koller, S. H., LoBianco, A. C., Hutz, C. S., Bueno, J. L. O. \& Guedes, M. C. (2002). Avaliação de periódicos científicos brasileiros da área de psicologia. Ciência da Informação, 31, 163-177.

Recebido em 01/11/2011

Reformulado em 22/11/2011

Aprovado em 02/12/2011 
Sobre os autores:

Lariana Paula Pinto é psicóloga, mestre e doutoranda em Psicologia pelo Programa de Pós-Graduação Stricto Sensu em Psicologia da Universidade São Francisco - USF.

Thatiana Helena de Lima é psicóloga, mestre e doutoranda em Psicologia pelo Programa de Pós-Graduação Stricto Sensu da Universidade São Francisco - USF. Bolsista CAPES.

Robisom Carlos de Lima é graduando em Psicologia pela Universidade São Francisco - Campus Itatiba e bolsista do Programa de Iniciação Científica PIBIC/CNPq, trabalhando na área da Avaliação Psicológica. 